\title{
Nutritionat of Metabolism
}

Abad, L. 223

Abid, Z.B. 216

Adeli, K. 208

Adler, S. 42

Agrawal, B.K. 477

Aguilar, M.V. 402

Aguirre, C.A. 482

Akan, P. 53

Akin, F. 557

Aksnes, L. 146

Aksungar, F.B. 88

Akyildiz, M. 88

Alexander, J. 146

Al-Jazairi, M.I. 277

Aller, R. 134, 374, 416

Anděl, M. 461

Andrade Goulart, E.M. 35

Andrés, P. 367

Andrés-Lacueva, C. 493

Antal, M. 448, 454

Aparicio, A. 367

Arató, G. 448, 454

Ardalan, G. 208

Arnault, N. 139

Arrieta, F.J. 402

Ayerza, R., Jr. 27

Ayhan, Y. 331

Banz, W. 42

Bar-Hen, A. 139

Barkeling, B. 264

Barkoukis, H. 512

Barmada, M.M. 252

Bassil, M.S. 14

Bastemir, M. 557

Battelino, T. 439

Bediz, C. 53

Bertrais, S. 139

Biró, L. 448, 454

Bissoli, L. 75

Bjornsson, S. 527

Bocquet, M. 421

Boesch-Saadatmandi, C. 395

Boev, M. 82

Bokor, S. 550

Bose, K.S.C. 477

Bosello, O. 75

Branca, F. 108, 115

Brandsch, C. 387

Brantsæter, A.L. 146

Bray, G.A. 163

Breen, P.A. 182
Brehme, U. 115

Bruno, G. 571

Bukan, N. 22

Burden, V.R. 182

Cak1, S. 331

Calder, P.C. 155

Callahan, H.S. 182

Campos Queiroz, L. 35

Cancelli, F. 75

Caroselli, C. 571

Casajús, J.A. 288

CASPIAN Study Group 208

Castagno, E. 575

Chen, J. 258

Cho, K.W. 42

Choi, J.-H. 119

Choi, S.-W. 119

Christophe, A. 541

Cinasal, G. 22

Coates, W. 27

Conde, R. 134, 374, 416

Cuervo, M. 115

Dalgıç, N. 22

Damini, R. 244

Davis, J. 42

de Campos Machado, L.J. 35

de Luis, D.A. 416

De Henauw, S. 172

de Luis, D.A. 134, 374

Declercq, D. 541

Decsi, T. 550

Delanghe, J. 172

Delavari, A. 208

Demmelmair, H. 433

Di Francesco, V. 75

Díaz, E.O. 482

Díaz, L.E. 359

Dlouhý, P. 461

Dolunay, G. 331

Doncheva, N. 82

Donmez, M. 331

Duan, Q. 270

Dueñas, A. 223

Duperray, B. 139

Eder, K. 387

Egli, I.M. 115

Elmadfa, I. 115, 324

Ergenekon, E. 22

Erguven, M. 331

Estruch, R. 493
Feki, M. 216

Ferrell, R.E. 252

Fidler Mis, N. 439

Fingerlin, T.E. 252

Freisling, H. 499

Fuhrmann, H. 155

Galan, P. 139

Galgani, J.E. 482

Ganel, U. 468

Gedrich, K. 563

Gelecek, N. 53

Glezer, I. 468

Gonzalez Sagrado, M. 134, 416

González Sagrado, M. 374

González, M.J. 402

González-Gross, M. 359

Greco, D.B. 35

Gronowska-Senger, A. 115

Gruppo Italiano Studio Sibutramina 75

Guerra, A. 433

Guihard-Costa, A.-M. 421

Guimarães Penido, M. 35

Haber, P. 428

Hagve, T.-A. 146

Hahn, A. 335

Hailer, S. 563

Halleen, J. 65

Hamdaoui, M.H. 216

Hasanoğlu, A. 22

Haugen, M. 146

Hédhili, A. 216

Hellstrom, P.M. 264

Hercberg, S. 139

Hien, V.T.T. 7

Higginbotham, A. 42

Higuchi, T. 281

Hirche, F. 387

Hong, H.-R. 197

Hop, L.T. 7

Hornig, D.H. 301

Hoybye, C. 264

Hrnčířová, D. 461

Huang, F. 258

Hursting, S.D. 232

Hwalla, N.C. 14

Hwang, Y.-H. 126

Izaola, O. 134, 374, 416

Izquierdo-Pulido, M. 204, 493
Jang, H.-S. 119

Jing, M.-Y. 345

Jonsson, P.V. 527

Julshamn, K. 146

Justo, I. 223

Kamau-Mbuthia, E. 324

Kang, H.-S. 197

Kang, K.-J. 126

Kang, M.Y. 519

Kaptanoglu, B. 557

Kassu, A. 7

Kelishadi, R. 208

Kesse-Guyot, E. 139

Khan, N.C. 7

Khanh, N.L.B. 7

Kiener, C. 352

Kim, C.E. 519

Kim, K.-R. 119

Kim, M.H. 533

Kim, S.-J. 379

Kim, W.Y. 533

Kim, Y.-C. 42

Kirwan, J.P. 512

Knerr, I. 352

Koç, E. 22

Koc, S. 331

Koletzko, B. 433, 550

Konstantynowicz, J. 574

Koren, M.S. 182

Korpela, R. 65

Koschizke, J.W. 335

Kotnik, P. 439

Kozan, O. 53

Krishnan, R.K. 512

Križišnik, C. 439

Kung, A.W.C. 59

Lala, R. 228, 575

Lam, N.T. 7

Lamuela-Raventós, R.M. 493

Lane, M.A. 232

Lee, H.S. 533

Lee, J. 197

Lee, Y.R. 519

Legaz, A. 288

Leitzmann, C. 335

Leiva, M.T. 288

Leung, J.Y.Y. 59

Libanore, V. 228

Lien, D.T.K. 7

Linseisen, J. 563

López-Sobaler, A.M. 367 
Lu, T. 270

Lu, Z. 258

Machagna, M. 468

Maggini, S. 301

Mahmood-Arabi, M.S. 208

Majdzadeh, R. 208

Malindretos, P. 471

Manara, F. 571

Mansour, A.A. 277

Marchetti, C.M. 512

Marcos, A. 359

Marhol, P. 461

Marinaro, L. 228

Martínez, J.A. 115

Martínez-Para, M.C. 402

Martos, É. 448, 454

Mata-Bilbao, M.L. 493

Mateos, C.J. 402

Matthys, C.C. 182

Mavromichalis, I. 471

Maxwell, C. 188

Mazón, M.A. 223

Mazzali, G. 75

Meeuws, K.E. 182

Meltzer, H.M. 146

Meseguer, I. 402

Miles, E.A. 155

Miniero, R. 228

Minniti, A. 75

Monti, G. 228, 575

Moreira Guimarães, M.M. 35

Moreno, L.A. 288

Moss, L. 252

Most, E. 395

Most, M. 163

Motaghian, M. 208

Motkowski, R. 574

Moustaid-Moussa, N. 42

Mrayati, M.M. 14

Musumeci, S. 244

Nagy, K. 448, 454

Nakamori, M. 7

Nam, S.H. 519

Namazi, R. 208

Narva, M. 65

Naslund, E. 264

Navas-Carretero, S. 204

Navia, B. 367
Nelson, T.L. 252

Nhung, B.T. 7

Niagolov, Y. 82

Nimptsch, K. 563

Nolan, B. 512

Norberto, L. 244

Norris, J.M. 252

Nova, E. 359

O’Connor, T. 42

Obeid, O.A. 14

Orenshtein, A. 468

Ortega, R.M. 367

Ozdirenc, M. 53

Paoletti, M.G. 244

Papandreou, D. 471

Park, E. 1

Park, H.J. 533

Park, T.-G. 197

Pavlovic, M. 108

Penkov, A. 82

Pérez-Castrillón, J.L. 223, 374

Pérez-Granados, A.M. 204

Perkins, S.N. 232

Péter, S. 448, 454

Peterson, R. 42

Pinar, L. 53

Pineau, J.-C. 421

Pokorný, R. 461

Popov, B. 82

Prentice, A. 108

Press, Y. 468

Procházka, B. 461

Purnell, J.Q. 182

Pynaert, I. 172

Qian, L.-C. 345

Rambousková, J. 461

Ramel, A. 527

Ramezani, M.A. 208

Rascher, W. 352

Rasmussen, S.E. 146

Rauh, M. 352

Redmann, S. 163

Regöly-Mérei, A. 448, 454

Riazi, M.M. 208

Ribeiro de Oliveira Júnior, A. 35
Rissanen, J. 65

Robberecht, E. 541

Rodríguez-Rodríguez, E. 367

Rohrmann, S. 563

Romeo, J. 359

Romero, E. 416

Rood, J. 163

Roura, E. 493

Rousso, I. 471

Rudoy, I. 468

Saavedra, P. 402

Sacco, T. 75

Salanitri, T. 75

San Miguel, A. 223

Sanz, A. 223

Sarriá, B. 204

Savino, F. 228, 575

Schoppen, S. 204

Schweigert, F.J. 99

Shay, N. 42

Shimizu, E. 281

Shin, M.-J. 1

Shirai, N. 281

Shukla, A. 387

Širca-Čampa, A 439

Sistrun, S.N. 512

Smith, N.C.P. 232

Smith, S.R. 163

Smole, K. 439

Spielmann, J. 387

Spreitzer, A. 428

Stamou, M. 471

Stangl, G.I. 387

Štimec, M. 439

Strasser, B. 428

Ströhle, A. 335

Sun, J.-Y. 345

Sun, W. 345

Suzuki, H. 281

Szabó, C. 448, 454

Tam, S. 59

Tebbe, A. 42

Temmerman, M. 172

Teoman, N. 53

Tetens, I. 115

Thorén, M. 264

Thorsdottir, I. 108, 527
Topkaya, A.E. 88

Toschke, A.M. 433

Tümer, L. 22

Uauy, R.D. 482

Väänänen, K. 65

Vámos, A. 448

Van Biervliet, J.-P. 541

Van Biervliet, S. 541

Vanbillemont, G. 541

Vapaatalo, H. 65

Vaquero, M.P. 204

Vega, G. 223

Velcheva, A. 82

Villarroya, A. 288

Volkov, I. 468

Volpe, S.L. 188

Waldmann, A. 335

Wang, J. 258

Wang, X. 270

Wärnberg, J. 359

Wat, W.Z.M. 59

Waterhouse, A.L. 493

Weigand, E. 395

Weigel, C. 352

Weigle, D.S. 182

Weng, X.-Y. 345

West, A.L. 155

Wiererová, O. 461

Williams, E.A. 232

Wintergerst, E.S. 301

Wolfram, G. 108, 563

Woo, M.-H. 119

Wu, M. 270

Wu, X. 258

Yamamoto, S. 7

Yllmaz, O. 331

Yoshikawa, T. 408

Zamboni, M. 75

Zhang, C.G. 379

Zhang, G. 281

Zi, N.-T. 345

Zoico, E. 75

Zupančič, M. 439 